\title{
Straßengüterverkehr: geplantes EU-Entsenderecht - ein protektionistischer Affront?
}

\author{
Der Straßengüterverkehr ist das Rückgrat des Warenaustauschs im Europäischen \\ Binnenmarkt, denn der überwiegende Teil der in Europa gehandelten Waren wird allen \\ politischen Bekenntnissen zur Verlagerung auf die Schiene zum Trotz nach wie vor per Lkw \\ transportiert. In dieser Branche kommt es jedoch, auch angesichts des starken Wachstums \\ der letzten Jahre, zunehmend zu Verwerfungen. Vordergründig wird über das Problem des \\ Fahrermangels diskutiert, der sich nicht nur in Westeuropa, sondern mittlerweile sogar in \\ den osteuropäischen Staaten bemerkbar macht. Im Hintergrund stellt sich aber die Frage, \\ ob die Prinzipien des Binnenmarkts - insbesondere die Dienstleistungsfreiheit - und die \\ Wettbewerbssituation auf den nationalen Transportmärkten noch kompatibel sind.
}

Die westeuropäischen und speziell die deutschen Straßengüterverkehrsunternehmer verlieren seit langem Marktanteile auf den Transportmärkten, da sie mit den in der Regel günstigeren Anbietern aus Osteuropa zumindest preislich oft nicht mithalten können. Nach der Mautstatistik des Bundesamts für Güterverkehr betrug der Marktanteil deutscher mautpflichtiger Lastkraftwagen auf den Bundesautobahnen 2018 noch $55 \%$ - Tendenz fallend. ${ }^{1}$ Die Preisunterschiede am Markt basieren im Wesentlichen auf Kostendifferenzen beim Fahrpersonal, da auch die osteuropäischen Unternehmen im Schwerverkehr heute mit modernstem Gerät unterwegs sind und ohnehin alle Fahrzeugflotten angesichts der verfügbaren Tankkapazitäten länderübergreifend optimierte Tankstrategien verfolgen. Einzig beim Fahrpersonal schlägt das Lohngefälle zuungunsten der deutschen Fahrer voll durch. Die Verwerfungen auf dem Markt betreffen dabei nicht nur den grenzüberschreitenden und den Transitverkehr, sondern zunehmend auch den rein nationalen Verkehr, weil eine aktuell kaum zu handhabende und zu kontrollierende europäische Kabotageregelung Grauzonen für ausländische Anbieter (auch) auf dem deutschen Markt eröffnet hat.

Um das deutsche Transportgewerbe vor der Konkurrenz aus Osteuropa zu schützen, wurde der 2015 in Deutschland eingeführte Mindestlohn auch auf ausländische Lkw-Fahrer übertragen, sofern sie im Inland operieren. Nach Protest der EU-Kommission wurden später Transitfahrten von der Min-

(c) Der/die Autor(en) 2020. Open Access: Dieser Artikel wird unter der Creative Commons Namensnennung 4.0 International Lizenz (https:// creativecommons.org/licenses/by/4.0/deed.de) veröffentlicht.

Open Access wird durch die ZBW - Leibniz-Informationszentrum Wirtschaft gefördert.

1 Vgl. Bundesamt für Güterverkehr: Mautstatistik. Tabellen 2018, Köln 2019, S. 5, https://www.bag.bund.de/SharedDocs/Downloads/DE/ Statistik/Lkw-Maut/Jahrestab_17_18.html?nn=12716 (9.3.2020). destlohnregelung ausgenommen. Trotzdem leitete die Kommission ein Vertragsverletzungsverfahren gegen Deutschland, Frankreich und Österreich ein, da die Anwendung der Mindestlohnvorschriften auf ausländische Kraftfahrer in den betroffenen Ländern nach ihrer Einschätzung die Dienstleistungsfreiheit unverhältnismäßig einschränke. ${ }^{2}$

Das Problem wird durch die in der Vergangenheit national uneinheitlich umgesetzten Vorgaben der EU-Richtlinie zu Lenk- und Ruhezeiten verschärft. So kam es in Deutschland, Frankreich, Belgien und Italien zu einem Verbot, die regelmäßige Wochenruhezeit (45 Stunden nach 6 Arbeitstagen) in der Fahrerkabine zu verbringen. Man berief sich dabei auf ein entsprechendes Urteil des Europäischen Gerichtshofs

2 Vgl. U. Pötsch: Entsendung von Kraftfahrern. EU will Anwendbarkeit der Entsenderichtlinie im Transportgewerbe klären, cepAdhoc vom 2.7.2019, S. 4, https://www.cep.eu/fileadmin/user_upload/cep.eu/ Studien/cepAdhoc_Entsendung_von_Kraftfahrern/cepAdhoc_Entsendung_von_Kraftfahrern.pdf (9.3.2020).

Prof. Dr. Alexander Eisenkopf ist Inhaber des Zeppelin-Lehrstuhls für Wirtschafts- und Verkehrspolitik an der Zeppelin Universität Friedrichshafen.

Prof. Dr. Dr. h.c. Andreas Knorr ist Professor für Volkswirtschaftslehre, insbesondere für Wirtschaftsund Verkehrspolitik, an der Deutschen Universität für Verwaltungswissenschaften Speyer. 
(EuGH) vom 20.12.2017. ${ }^{3}$ Auf „kaltem Wege“ versuchte z.B. Dänemark dieses Phänomen zu unterbinden, indem zum 1.7.2018 eine Höchstparkdauer für Lkw auf Autobahnparkplätzen eingeführt wurde. Praktisch ist das Verbringen der 45-stündigen Pause in der Fahrerkabine bereits heute in den meisten EU-Mitgliedstaaten untersagt.

Angesichts dieser komplexen, sich zuspitzenden Gemengelage fehlt eine einheitliche, wirksame und zielführende Umsetzung sowohl der Entsenderichtlinie als auch der Kabotage- und Sozialvorschriften der EU. Bereits 2016 versprach die damalige EU-Verkehrskommissarin Violeta Bulc die Verabschiedung eines „Road Packages“, das aber als „Mobility Package“ angesichts der konfligierenden Interessen in diesem Bereich über Jahre kontrovers diskutiert wurde. Im Kern ging der Streit um die Regelungen in den Bereichen Entsendevorschriften und Kabotage sowie um die Neujustierung der Vorschriften zu den gesetzlichen Lenk- und Ruhezeiten.

Entgegen den Erwartungen gelang dem Europäischen Parlament im April 2019 die Einigung auf eine gemeinsame Position zum Mobilitätspaket. Auch der Rat der Verkehrsminister hatte sich im Dezember 2018 entsprechend positioniert, wobei die einzelnen Festlegungen im Detail jedoch noch nicht vollständig mit dem Richtlinienvorschlag der EU-Kommission vom 31.5.2017 kommensurabel waren. ${ }^{4}$ Trotzdem ist es Ende 2019 gelungen, das Vorhaben im üblichen Trialogprozess voranzubringen und einen Kompromiss für ein Mobilitätspaket zu verhandeln, der im Dezember 2019 durch eine qualifizierte Mehrheit im Ministerrat verabschiedet wurde. ${ }^{5}$ Diesen hat der Verkehrsausschuss des EU-Parlaments am 21.1.2020 gebilligt, sodass einer finalen Zustimmung des Plenums kaum etwas im Wege stehen dürfte.

Da das derzeitige Durch- und Nebeneinander mehr oder weniger restriktiver nationaler Regelwerke ökonomisch und politisch völlig unbefriedigend ist, ist positiv anzumerken, dass man einer einheitlichen EU-weiten Regelung zumindest näher gekommen ist. Entsende- und Kabotageregelungen sind ein zentraler Baustein des institutionellen Rahmens

3 Vgl. Europäische Gerichtshof: Im Straßentransportsektor dürfen die Fahrer die innen zustehende regelmäßige wöchentliche Ruhezeit nicht in ihrem Fahrzeug verbringen, Pressemitteilung vom 20.12.2017, Nr. 145/17, https://curia.europa.eu/jcms/upload/docs/application/ pdf/2017-12/cp170145de.pdf (9.3.2020).

4 Vgl. Europäische Kommission: Vorschlag für eine Richtlinie des Europäischen Parlaments und des Rates zur Änderung der Richtlinie 2006/22/EG bezüglich der Durchsetzungsanforderungen und zur Festlegung spezifischer Regeln im Zusammenhang mit der Richtlinie 96/71/EG und der Richtlinie 2014/67/EU für die Entsendung von Kraftfahrern im Straßenverkehrssektor, Brüssel, 31.5.2017, COM(2017) 278 final, https://ec.europa.eu/transparency/regdoc/rep/1/2017/DE/ COM-2017-278-F1-DE-MAIN-PART-1.PDF (9.3.2020).

5 Vgl. F. Hütten: EU-Mobilitätspaket nimmt Hürde im Ministerrat, in: Deutsche Verkehrs-Zeitung (DVZ) vom 20.12.2019. für Marktstruktur und Wettbewerb auf dem Straßengüterverkehrsmarkt und beeinflussen das Laderaumangebot und die Fahrerkapazitäten auf den nationalen Märkten in nicht unerheblichem Maße.

Es gibt jedoch Anlass zu schwerwiegenden Bedenken hinsichtlich des Umfangs und Inhalts der geplanten Regelungen, da hierdurch der Wettbewerb im Straßengüterverkehrsgewerbe unnötig beschränkt, die Dienstleistungsfreiheit im Europäischen Binnenmarkt zum Teil effektiv ausgehebelt und protektionistischen Tendenzen Vorschub geleistet wird. Bedauerlicherweise hat sich die Bundesregierung im Koalitionsvertrag für die laufende 19. Legislaturperiode nicht eindeutig für eine wettbewerbliche Lösung der Entsendeproblematik ausgesprochen und unterstützt die aktuelle Beschlusslage auf EU-Ebene. ${ }^{6}$

\section{Allgemeine Entsenderichtlinie}

Ende Mai 2018 verabschiedete das EU-Parlament mit großer Mehrheit eine Reform der allgemeinen EU-Entsenderichtlinie, nachdem sich bereits Ende Februar 2018 die Verhandlungsführer von Kommission, Rat und Parlament auf eine gemeinsame Linie geeinigt hatten. ${ }^{7}$ Die Initiative zur Reform der auf das Jahr 1996 zurückgehenden und zuletzt 2014 revidierten Entsenderichtlinie wurde von der EU-Kommission eingebracht. Sie hatte sich in ihren politischen Leitlinien verpflichtet, den Grundsatz gleichen Lohns für gleiche Arbeit am gleichen Ort zu fördern und dieses Projekt in ihr Arbeitsproramm 2016 aufgenommen. Ziel dieser Reform war aus Sicht der EU-Kommission, die Entsendung von Arbeitnehmern zu erleichtern, dabei aber gerechte Entlohnungsund gleiche Wettbewerbsbedingungen für entsendende wie lokale Unternehmen im Aufnahmeland zu gewährleisten.

Bisher sah die EU-Entsenderichtlinie 96/71/EG nur einen Mindeststandard vor; für entsandte Arbeitnehmer muss im jeweiligen Aufnahmeland z. B. der jeweilige gesetzliche Mindestlohn gezahlt werden. Im Zuge der Neuregelung wird entsandten Arbeitnehmern jedoch ein Rechtsanspruch auf

6 "Sozialbetrug und Sozialdumping darf es auf unseren Straßen nicht geben. Wir werden daher klarere Regelungen der Haftung entlang der Logistikkette entwickeln. In diesem Zusammenhang werden wir auch die EU-Initiative „Europe on the Move“ kritisch begleiten. Wichtige Kriterien dabei sind die Anwendung der Entsenderichtlinie für Lkw-Fahrer und der Mindestlohnregelungen. Die Kabotage darf dabei nicht weiter ausgeweitet werden. Wir werden wirksame Instrumente zur Kontrolle der Kabotage schaffen.“, vgl. Koalitionsvertrag zwischen CDU, CSU und SPD: Ein neuer Aufbruch für Europa. Eine neue Dynamik für Deutschland. Ein neuer Zusammenhalt für unser Land, 19. Legislaturperiode, Berlin 2018, Zeile 3815 ff., https://www.cdu.de/system/tdf/ media/dokumente/koalitionsvertrag_2018.pdf?file=1 (9.3.2020).

7 Europäisches Parlament, Europäischer Rat: Richtlinie (EU) 2018/957 des Europäischen Parlaments und des Rates vom 28.6.2018 zur Änderung der Richtlinie 96/71/EG über die Entsendung von Arbeitnehmern im Rahmen der Erbringung von Dienstleistungen, https://eur-lex.europa.eu/legal-content/DE/TXT/PDF/?uri=CELEX:32018L0957 (9.3.2020). 
die Zahlung der üblichen Tariflöhne zugestanden, falls allgemeinverbindliche Tarifverträge bestehen sowie auf alle Prämien, Zulagen und Urlaubsregelungen, die auch vergleichbaren einheimischen Arbeitnehmern zu gewähren sind (z. B. Weihnachtsgeld). Entsendungen werden zudem auf zwölf Monate begrenzt (mit einer Verlängerungsmöglichkeit auf 18 Monate). Weiterhin dürfen Reise-, Verpflegungs- und Unterbringungskosten nicht mehr vom Arbeitnehmerlohn abgezogen werden, und die Arbeitgeber haben für angemessene Unterbringungsbedingungen zu sorgen, die den jeweiligen nationalen Vorschriften entsprechen. Die EU-Mitgliedstaaten haben die Neuregelungen bis Ende Juni 2020 umzusetzen. Dies ist in Deutschland aktuell noch nicht passiert; derzeit wird ein entsprechender Referentenentwurf des Bundesministeriums für Arbeit und Soziales diskutiert.

\section{Entsenderichtlinie und Straßengüterverkehr}

Ursprünglich sollte nach den Vorstellungen der EU-Kommission die überarbeitete Entsenderichtlinie grundsätzlich auch für den Verkehrssektor gelten, was bedeuten würde, dass ausländische Fahrer im Straßengüterverkehr in jedem durchfahrenen Land für den dort zurückgelegten Streckenabschnitt entsprechend dem landesüblichen Lohn bezahlt werden müssten. Im Straßengüterverkehrsgewerbe sind jedoch entsandte Arbeitnehmer regelmäßig nur für deutlich kürzere Zeit als in anderen Wirtschaftszweigen in einem anderen EU-Mitgliedstaat tätig. Daher hatte die EU-Kommission in ihrem sogenannten 1. Mobilitätspaket modifizierte Reformvorschläge vorgelegt, die insbesondere im EU-Parlament kontrovers diskutiert wurden.

Sie sahen vor, dass bei sogenannten grenzüberschreitenden Beförderungen im weiteren Sinne die Entsenderichtlinie anzuwenden sei, allerdings nicht hinsichtlich der Vorschriften über Löhne und bezahlten Mindestjahresurlaub. Diese Ausnahme sollte allerdings bei einem Aufenthalt im Aufnahmeland von mehr als drei Tagen innerhalb eines Kalendermonats entfallen. Für Kabotageverkehre sollte die Entsenderichtlinie dagegen umfassend anwendbar sein, für reine Transitverkehre demgegenüber nicht. ${ }^{8}$

Nach intensiven Diskussionen und einer längeren Hängepartie schloss sich das Parlament Anfang April 2018 hinsichtlich der Transit- und Kabotageverkehre mit Einschränkungen der Position der EU-Kommission an. Darüber hinaus machte der Verkehrsausschuss des EUParlaments am 4.6.2018 einen eigenständigen, sehr restriktiven Vorschlag zur Neuregelung der Kabotage. ${ }^{9}$ Obwohl dieser Regelungsvorschlag in den meisten Me-

8 Vgl. Europäische Kommission, a.a.O.

9 Vgl. zu Details A. Gieße: Sozialvorschriften für Fahrer im Fokus, Verkehrsrundschau, Nr. 24, 2018, S. 15. dien als Liberalisierung der bisherigen „Drei in sieben“Vorschrift qualifiziert wurde, blieb er weit hinter den Plänen der Kommission zurück. Sie wollte zwar die Frist für Kabotagebeförderungen von sieben auf fünf Tage verkürzen, im Gegenzug aber jegliche sonstige Beschränkungen streichen. Das bisherige Regime, das sich in der Praxis als kaum effektiv durchsetzbar erwiesen hatte, sollte damit zugunsten einer wettbewerbsfreundlichen und besser kontrollierbaren Regelung verändert werden.

In der Folgezeit bildeten sich für die Kabotageregelungen und Sozialvorschriften (bezüglich der Lenk- und Ruhezeiten) bei Parlament und beim EU-Rat der Verkehrsminister klare Verhandlungspositionen heraus. Das EU-Parlament wollte die existierende Regelung durch eine Drei-TagesFrist ersetzen, um eine besser kontrollierbare Regelung zu erreichen. Gefordert wurde zudem die Registrierung von Grenzübergängen durch Fahrtenschreiber sowie ein „Karenzzeitraum“ (Cooling-off-Phase) von 60 Stunden Aufenthalt im Heimatland, bevor weitere Kabotagefahrten mit demselben Fahrzeug durchgeführt werden dürfen. Demgegenüber wollte der Rat die bisher geltende Kabotageregelung beibehalten und durch eine anschließende fünftägige Karenzzeit beschränken.

In den Verhandlungen zwischen Parlament und Ministerrat im Dezember 2019 hat sich der Rat mit dieser Forderung durchgesetzt. Die bestehende „Drei in sieben“Kabotageregelung wird beibehalten und soll durch eine viertägige Cooling-off-Phase zusätzlich verschärft werden. Eine Beschränkung der Kabotage soll sogar im Kombinierten Verkehr möglich sein. Zwar sieht die EUKommission die geplanten Neuregelungen auch aus der Perspektive des Klimaschutzes kritisch, doch will sie das seit zweieinhalb Jahren diskutierte Richtlinienpaket nicht mehr grundsätzlich blockieren. ${ }^{10}$

Neben der vorgeschriebenen wöchentlichen Ruhezeit müssen die Fahrer in regelmäßigen Abständen (mindestens alle drei Wochen) nach Hause zurückkehren; außerdem müssen die Fahrzeuge spätestens alle acht Wochen in das Land ihrer Niederlassung zurückkommen. Auch diese Regelungen sind gegenüber dem vorherigen Diskussionsstand restriktiver. ${ }^{11}$ Hinsichtlich der Anwendung der Entsenderichtlinie ist vorgesehen, dass diese vollumfänglich bereits ab dem ersten Tag gelten soll, mit Ausnahmen des reinen Transits oder bilateraler Verkehre mit maximal zwei Be- oder Entladevorgängen. Zudem gelten die Marktzugangsvoraussetzungen sowie die Regelungen

10 Vgl. F. Hütten, a.a.O.

11 Vgl. o. V.: Endlich Einigung beim ersten EU-Mobilitätspaket, Verkehrsrundschau online vom 12.12.2019, https://www.verkehrsrundschau. de/nachrichten/endlich-einigung-beim-ersten-eu-mobilitaetspaket-2518204.html (9.3.2020). 
zu Lenk- und Ruhezeiten im internationalen Verkehr jetzt auch für Fahrzeuge über 2,5 t zulässige Gesamtmasse.

\section{Fata Morgana „gleicher Wettbewerbsbedingungen“}

Das deutsche Straßengüterverkehrsgewerbe begrüßt eine Verschärfung der Entsende- und Kabotageregelungen sowie der Anwendung der Sozialvorschriften nachdrücklich. ${ }^{12}$ Sowohl die Entscheidungen auf EU-Ebene als auch die Positionen der Verbände sind allerdings aus ökonomischer Perspektive kritisch zu hinterfragen. Häufig ist in der Diskussion über die Entsenderegeln für grenzüberschreitend oder im Rahmen der Kabotage tätig werdende Lkw-Fahrer von „Lohndumping“ oder „unfairem Wettbewerb“ die Rede. Der Begriff „Lohndumping“ wird dann dahingehend substanziiert, dass für gleiche Arbeit am gleichen Ort der gleiche Lohn zu zahlen sei; ansonsten liege eine Wettbewerbsverzerrung vor. Um fairen Wettbewerb zu gewährleisten, seien „gleiche Wettbewerbsbedingungen" für inländische Unternehmen wie Dienstleistungsanbieter aus dem Ausland herzustellen.

Während die Forderung nach gleichem Lohn für gleiche Arbeit am gleichen Ort direkt das menschliche Gerechtigkeitsempfinden anspricht und damit gefühlsmäßig meistens unmittelbare Zustimmung erheischt, sollte klar sein, dass es einen funktionsfähigen Wettbewerb bei „gleichen“ Wettbewerbsbedingungen sachlogisch gar nicht geben kann, da bei vollkommen gleichen Bedingungen für alle Marktteilnehmer kein Wettbewerb stattfinden wird. Es kann folglich ökonomisch nur darum gehen, bestimmte Verzerrungen des Wettbewerbs, z. B. aufgrund staatlicher Eingriffe in den Markt, zu korrigieren bzw. zu verhindern. Unterschiedliche Faktorproduktivitäten und wirtschaftliche Entwicklungsstandards, die sich letztlich in unterschiedlichen Löhnen bzw. Lohnniveaus äußern, sind gerade eine Triebfeder des wirtschaftlichen Austauschs in einem Wettbewerbsumfeld.

Eine politik-induzierte Ex-ante-Harmonisierung der Wettbewerbsbedingungen wie sie sich z. B. in der Idee äußert, auch für zeitweilig entsandte Arbeitnehmer aus dem Ausland den nationalen Tariflohn zu zahlen, torpediert damit den Wettbewerb privater Akteure und die daraus resultierenden Wohlstandsgewinne sowie die Wohlfahrtsgewinne aus dem institutionellen Wettbewerb. Als seminaler Anwendungsfall solcher wettbewerbsfeindlicher Regelungen ist die in den 1990er Jahren in Deutschland entwickelte Entsenderichtlinie für die Bauwirtschaft anzusehen. Sie sollte sicherstellen, dass Bauarbeiter aus Südeuropa in Deutschland nur zu gleichen Bedingungen angestellt wer-

12 Vgl. etwa Bundesverband Güterverkehr und Logistik: BGL zieht überwiegend positive Bilanz aus den Beschlüssen des EU-Verkehrsministerrats zum Mobilitätspaket, Pressemitteilung vom 4.12.18, https://www.bgl-ev.de/web/medien/presse/article_archiv. htm\&news $=3310$ \&year=2018 (9.3.2020). den dürfen wie einheimische. Damit wurde faktisch der inländische Tariflohn zum Mindestlohn für Arbeitnehmer aus dem Ausland erklärt, wodurch die inländischen Arbeitnehmer wirksam vor Konkurrenz geschützt sind..$^{13}$

\section{Entsenderichtlinie als Musterbeispiel protektionistischer Außenhandelspolitik}

Analysiert man diese Problematik aus außenhandelstheoretischer Sicht, ist festzuhalten, dass jeder Warenexport bzw. -import ökonomisch zwingend auch den Export bzw. Import der in die Warenproduktion eingeflossenen Faktorleistungen bedeutet, nicht zuletzt der Arbeitsleistung. Auch im Warenhandel konkurrieren somit inländische Arbeitskräfte unmittelbar mit ausländischen, wenngleich dies für die betroffenen inländischen Arbeitnehmer wie auch für die inländischen Nachfrager von Importgütern weit weniger transparent ist.

Fast alle Staaten (wie auch die EU selbst) betreiben allerdings eine tendenziell protektionistische - sprich merkantilistisch orientierte - Außenhandelspolitik; in diesem Zusammenhang wird in der Regel, und dies nicht zuletzt in Deutschland, der Warenhandel fälschlicherweise als ökonomisch wichtiger wahrgenommen als der Dienstleistungsaustausch. Das populäre politische Ziel möglichst hoher Exportüberschüsse im Warenverkehr (Handelsbilanz) steht ökonomisch allerdings auf tönernen Füßen, denn es steht nicht nur in fundamentalem Widerspruch zum gesamtwirtschaftlichen Ziel des im Stabilitäts- und Wachstumsgesetz von 1968 verankerten „außenwirtschaftlichen Gleichgewichts". Übersehen wird dabei auch, dass die Exportüberschüsse eines Landes zwingend mit entsprechenden Importüberschüssen der Zielländer der einheimischen Exportgüter einhergehen müssen. Zu beachten ist folglich auch, dass Exportüberschüsse zugleich immer auch (Netto-) Kapitalexporte implizieren: Investitionen erfolgen aufgrund der Kapitalabflüsse im Ausland anstatt im Inland bzw. es kommt zur Akkumulation von finanziellen Anspruchstiteln, also (Netto-)Forderungen, gegenüber dem Ausland, deren langfristige Werthaltigkeit häufig infrage zu stellen ist.

Ein zur Warenbilanz gegenläufiger Saldo in der Dienstleistungsbilanz - z. B. durch den (Netto-)Import von Transportdienstleistungen - stellt damit eine volkswirtschaftlich wichtige Möglichkeit dar, fundamentale Zahlungsbilanzungleichgewichte und die mit innen zwangsläufig auftretenden makroökonomischen Verwerfungen zumindest abzumildern. Dies gilt insbesondere angesichts der Tatsache, dass seit Einführung der EU-Währungsunion

13 Vgl. W. Eichhorst: Gleicher Lohn für gleiche Arbeit am gleichen Ort? Die Entsendung von Arbeitnehmern in der Europäischen Union, in: Zeitschrift für Arbeitsmarktforschung (ZAF), 38. Jg. (2005), H. 2-3, S. 209 ff. 
der Ausgleich von Zahlungsbilanzungleichgewichten zwischen den Mitgliedstaaten der Eurozone durch entsprechende Wechselkursanpassungen nicht mehr möglich ist. Weiterhin gilt, dass ohne flankierende Transportdienstleistungen grundsätzlich weder Warenproduktion noch Warenhandel möglich ist. Das bedeutet wiederum, dass die Wettbewerbsfähigkeit deutscher Warenexporteure (Handelsbilanz) auch ganz wesentlich vom Preis, der Produktivität und der Verfügbarkeit von Transportdienstleistungen abhängig ist. Ein chronischer Fahrermangel bzw. protektionistisch motivierte Beschränkungen des ausländischen Fahrerangebots im Inland wirken damit nicht nur nachteilig für das deutsche Transportgewerbe, sondern schaden auch der deutschen (Waren-)Exportwirtschaft.

Die Erbringung von Transportdienstleistungen geht aber grundsätzlich immer mit der temporären Migration der Besatzungen (Fahrer, Piloten etc.) einher; dies gilt sowohl für rein innerstaatliche als auch für ein- und ausgehende grenzüberschreitende Transporte. Folgt man nun argumentativ konsequent der politischen und gewerkschaftlichen Logik des einheitlichen Lohnregimes für in Deutschland temporär tätige ausländische Fahrer, müsste spiegelbildlich auch akzeptiert werden, dass deutsche Fahrer bei Auslandsfahrten zu den dort üblichen - nach oben oder unten abweichenden - Konditionen entlohnt werden. Letztlich wirkt das von der allgemeinen Entsenderichtlinie verfolgte Lohnregulierungsregime somit dem im Falle offener Dienstleistungs- und Arbeitsmärkte (zumindest tendenziell) erfolgenden Ausgleich der Faktorpreise entgegen. Es handelt sich bei einer solchen Entsenderichtlinie daher um einen wettbewerbsfeindlichen Ansatz protektionistischer Außenhandelspolitik, der die internationale Integration der Arbeitsmärkte verzögert bzw. verhindert.

\section{Empfehlungen zur Regulierung internationaler Aktivitäten von Güterkraftverkehrsunternehmen}

Diese Überlegungen gelten umso mehr für das Transportgewerbe, wo echter Dienstleistungswettbewerb einen umfassenden Marktzugang auch zu Kabotageverkehren erfordert, ohne sofort Lohnkostenvorteile ausländischer Unternehmen durch die Vorgabe nationaler Tariflöhne als Mindestlöhne zu kompensieren..$^{14}$ Daher sollte aus wohlfahrtsökonomischer Sicht eine völlige Freigabe der Kabotage im Straßengüterverkehr das Ziel der europäischen Verkehrspolitik sein, d. h. die Realisierung umfassender Dienstleistungsfreiheit im

14 Kritisch zur Erzwingung von tariflichen Mindestlöhnen für ausländische Arbeitnehmer, die im Inland beschäftigt werden, äußern sich auch T. Bauer, C. M. Schmidt: Mindestlöhne und das Entsendegesetz, in: Wirtschaftsdienst, 87. Jg. (2007), H. 3, S. 161, https://www. wirtschaftsdienst.eu/inhalt/jahr/2007/heft/3/beitrag/mindestloehneund-das-entsendegesetz.html (9.3.2020).
Verkehr, wie sie spätestens seit dem berühmten Binnenmarkturteil des EuGH vom 22. Mai 1985 gefordert wird. ${ }^{15}$

Lediglich aus sozialpolitischen Erwägungen oder im Sinne einer Übergangs- und Anpassungsregelung scheinen eine (schrittweise aufzulockernde) zeitliche Begrenzung und die Verpflichtung auf den nationalen Mindestlohn bei Kabotageverkehren motivierbar. Weiter gehende Eingriffe in den Markt verstoßen in eklatanter Weise gegen die Prinzipien des EUBinnenmarkts und dienen in erster Linie dem Schutz von Branchen- und Lobbyinteressen. Sie sind zudem vor dem Hintergrund von aktuell diskutierten Kapazitätsengpässen und Fahrermangel im deutschen Straßengüterverkehr als kontraproduktiv zu bezeichnen. Damit verfehlen die aktuell auf EU-Ebene geplanten Regelungen das Ziel einer effizienzorientierten Regulierung des Güterkraftverkehrssektors; hinzuweisen ist auch auf die erheblichen bürokratischen Nachweis- und Dokumentationspflichten der jetzt gefundenen Regelungen mit einer Cooling-off-Periode und periodischen Rückkehrpflichten der Lkw und der Fahrer. Belgien, das sich bei der Abstimmung der EU-Botschafter enthalten hat, hat bereits angekündigt, wegen der „Abkühlungsperiode" den EuGH anzurufen. ${ }^{16}$

Die ablehnende Reaktion des deutschen Güterkraftverkehrsgewerbes hinsichtlich einer Lockerung der Kabotage ist aus der Branchenperspektive nachvollziehbar. Es geht um das Wachstum des eigenen Gewerbes, um dessen Gewinne und Arbeitsplätze. Umgekehrt widersetzen sich viele osteuropäische Staaten den geplanten Regelungen, sind aber im Ministerrat mit qualifizierter Mehrheit überstimmt worden. ${ }^{17}$ Das bulgarische Parlament hat sogar vor geraumer Zeit einstimmig eine Entschließung angenommen, welche die Regierung auffordert, die Interessen der bulgarischen Transporteure und Spediteure bei der EU zu verteidigen. Nach eigenen Angaben erwirtschaftet der Transport- und Speditionssektor $15 \%$ des bulgarischen Bruttoinlandsprodukts. ${ }^{18}$

In einem gemeinsamen Markt mit umfassender Dienstleistungsfreiheit wie der EU sollte der Schutz nationaler Partialinteressen nicht so weit gehen, dass über eine Entsenderegelung die nationalen Tarifstrukturen allgemeinverbindlich für alle Anbieter aus der EU erklärt werden und damit der Wettbewerb ausgeschaltet bzw. behindert wird. Es spricht vieles dafür, dass eine wettbewerbskonforme Regelung darin bestehen könnte, für die Kabota-

15 Vgl. G. Aberle: Transportwirtschaft. Einzelwirtschaftliche und gesamtwirtschaftliche Grundlagen, 4. Aufl., München, Wien 2009, S. 173 ff.

16 Vgl. F. Hütten, a.a. O.

17 Ebenda.

18 Vgl. o. V.: Sofia lehnt neue EU-Regeln gegen Lohndumping für Spediteure ab, Verkehrsrundschau online vom 14.5.2018, https://www.verkehrsrundschau.de/nachrichten/sofia-lehnt-neue-eu-regeln-gegenlohndumping-fuer-spediteure-ab-2163696.html (9.3.2020). 
geverkehre lediglich den geltenden gesetzlichen Mindestlohn verbindlich zu machen, keinesfalls aber die tatsächlich gezahlten tariflichen Löhne oder sonstige Tarifbedingungen. Damit bedeutet die geplante EU-Regelung eine unverhältnismäßige Einschränkung des Preiswettbewerbs und der Dienstleistungsfreiheit des EU-Binnenmarktes - auch wenn es derzeit für den Straßengüterverkehr in Deutschland (noch) keine allgemeinverbindlichen Tarifverträge gibt. ${ }^{19}$ Zudem möchte die Bundesregierung bei der Umsetzung der EU-Entsenderichtlinie jenseits der Allgemeinverbindlichkeit den Tarifparteien im Inland die Möglichkeit eröffnen, für Entsendungen entsprechende Entlohnungsvorschriften zu vereinbaren. ${ }^{20}$

Grenzüberschreitende (und selbstverständlich auch reine Transit-)Verkehre sind dagegen von der Entsenderichtlinie komplett auszunehmen. Es besteht bei diesen Verkehren kein hinreichender Bezug zum Wettbewerb auf den nationalen Transportmärkten im Aufnahmestaat. Dahingehend ist der Kompromiss vom Dezember 2019 zu begrüßen, auch wenn der bürokratische Aufwand durch die Beschränkung von Ladevorgängen inhaltlich problematisch erscheint und zusätzliche Transaktionskosten produziert. Bereits heute werden die Regelungen der Entsenderichtlinie von einzelnen EU-Mitgliedstaaten als bürokratische Schikanen zur Abwehr ausländischer Konkurrenz genutzt. Umfangreiche Dokumentations- und Nachweispflichten erhöhen die Transaktionskosten massiv und wirken wohlstandsmindernd und wettbewerbsschädlich. Sinn und Zweck einer EU-Entsenderichtlinie sollte der Abbau bürokratischer Hindernisse für den gemeinsamen Markt sein und nicht die nochmalige Erhöhung der Komplexität. Vertreter osteuropäischer Länder verstehen sogar die mögliche Anforderung an Lkw-Fahrer, alle drei Wochen für eine Ruhepause nach Hause zurückkehren zu müssen, als Verletzung des Rechts auf freie Bewegung innerhalb der EU.

Das Argument, bei einer Freigabe der Kabotage und der Beschränkung der Entsenderichtlinie auf die Anwendung des Mindestlohns für die Kabotage würde ein „unfairer“ Wettbewerb von Unternehmen insbesondere aus Osteuropa das deutsche Straßengüterverkehrsgewerbe in seiner Existenz bedrohen, führt in die Irre. Eine solche Verpflichtung wirkt umgekehrt sogar diskriminierend und verfälscht den Wettbewerb. Angesichts der sich abzeichnenden Kapazitätsengpässe und des bereits heute spürbaren Fahrermangels sollte die Wirtschafts- und Verkehrspolitik

19 Vgl. zu dieser Einschätzung auch U. Pötsch, a. a. O., S. 8.

20 Vgl. Bundesministerium für Arbeit und Soziales: Gleicher Lohn für gleiche Arbeit - für alle in Europa: Lohndumping verhindern. Ordnung auf dem Arbeitsmarkt sichern. Mobilität fair gestalten. Eckpunkte des BMAS zur Umsetzung der EU-Entsenderichtlinie vom Mai 2019, https:// www.bmas.de/SharedDocs/Downloads/DE/Thema-Arbeitsrecht/eckpunkte-entsenderichtlinie.pdf?__blob=publicationFile\&v=4 (9.3.2020). die Marktöffnung vorantreiben, um die Funktionsfähigkeit der infolge der internationalen Arbeitsteilung und Spezialisierung zunehmend entlang der grenzüberschreitenden Wertschöpfungsketten organisierten logistischen Systeme nachhaltig zu gewährleisten. Abschottung vor ausländischer Konkurrenz ist in einer Situation absehbarer Fahrerknappheit volkswirtschaftlich eindeutig das falsche Signal. Hinzu kommt die Überlegung, dass angesichts massiver und steigender Exportüberschüsse ein stärkerer Dienstleistungsimport dem gesamtwirtschaftlichen Gleichgewicht durchaus förderlich sein kann.

\section{Regulierung von Ruhezeiten und Parkplatzsituation}

In der medialen Diskussion wird zuweilen heftig darüber geklagt, dass die ausländischen Fahrer in Deutschland unter menschenunwürdigen Bedingungen arbeiten. Sie sind in der Regel mindestens drei Wochen und teilweise länger am Stück unterwegs, hausen am Wochenende unter (angeblich) primitivsten Bedingungen auf Autobahnparkplätzen und verzichten in dieser Zeit auf ein Familienleben. Dass ein solcher Arbeitsplatz trotzdem für viele Osteuropäer wirtschaftlich attraktiv ist, liegt vor allem daran, dass sie durch ihre Tätigkeit ein im Vergleich zu alternativen Erwerbsmöglichkeiten in ihren Heimatländern weit überdurchschnittliches Einkommen erzielen können. Besonders attraktiv sind in diesem Zusammenhang Lohnmodelle, die ein relativ geringes Fixum mit (zumindest teilweise) steuer- und sozialabgabefreien Spesen verbinden.

Diese Kalkulation geht jedoch nur auf, wenn die ausgezahlten Spesen nicht für von den Transit- oder Kabotageländern gesetzlich vorgeschriebene Übernachtungen auBerhalb des Lkw verwendet werden müssen, was die an Wochenenden auf den Autobahnparkplätzen zu beobachtende Armada ausländischer Lkw erklärt. Dieser Zustand erscheint aus unserer nationalen Wohlstandsperspektive beklagenswert, wird aber von den Fahrern selbst akzeptiert (die ja durchaus die Option haben, ihre Spesen für Hotelübernachtungen einzusetzen). Sie ziehen eine Beschäftigung in solchen internationalen Umläufen und den damit verbundenen Unannehmlichkeiten offensichtlich einer Beschäftigung zu Hause vor, was auch die sich inzwischen häufenden Berichte über Fahrermangel in ihren Ursprungsländern (z.B. Polen, Ungarn) erklären kann, obwohl das Lohnniveau dort mittlerweile ebenfalls stark gestiegen ist. Letztlich spielt für die Übernachtung in den Lkw auch eine wichtige Rolle, dass damit eine lückenlose Überwachung der Fracht gewährleistet ist, die bei zwangsweise angeordneten Übernachtungen in einem Hotel in dieser Form nicht möglich wäre. Das mit der Reform der Sozialvorschriften geplante Verbot, die längeren Wochenruhezeiten im Lkw zu verbringen, sollte daher überdacht werden. 
Probleme auf den Parkplätzen zeigen sich jedoch nicht nur an Wochenenden, sondern auch im alltäglichen Verkehrsgeschehen. Einschlägige Studien schätzen, dass in Deutschland über 30000 Lkw-Parkplätze an Autobahnen bzw. in Autobahnnähe fehlen. Dieses Problem ist nicht neu und auch nicht ausschließlich dem anhaltenden Verkehrswachstum geschuldet. Vielmehr zeigte es sich bereits nach dem Wirksamwerden der 2006 verabschiedeten europäischen Verordnung zu Lenk- und Ruhezeiten, die eine drastische Verschärfung der gesetzlichen Regelungen mit sich brachte. Allerdings hatte die Politik es versäumt, gleichzeitig auch für eine angemessene Erhöhung des Parkplatzangebots zu sorgen.

Zur chronischen Überlastung der Parkplätze haben aber nicht nur die Verschärfung der Lenk- und Ruhezeiten und das generelle Verkehrswachstum, sondern auch verschiedene andere Faktoren beigetragen. Die (gleichförmige) Optimierung logistischer Prozesse insbesondere im Inbound-Verkehr großer Industriebetriebe, im Handel und in der Hafenlogistik generieren Peak-Probleme mit geballten Parkproblematiken im Zulauf. Ein Lösungsansatz für eine effiziente Allokation könnte daher die Bepreisung des Parkens an der Autobahn für Lkw sein, anstatt die Kosten für Park- und Rastanlagen undifferenziert über die Wegekostenrechnung umzulegen. Damit würde der Knappheit von Parkraum Rechnung getragen, und es würde ein Anreiz für private Anbieter geschaffen, Parkraum auf Autohöfen nicht mehr umzuwidmen - was heute geschieht - bzw. die Kapazitäten zu erweitern. Es sollten vermehrt sichere, zertifizierte Lkw-Parkplätze ausgebaut werden, deren Nutzung bereits heute öffentlich gefördert wird.

Beiträge zu einer perspektivischen Lösung des Parkplatzproblems eröffnen auch Anwendungen für vollautonomes Fahren von Lkw auf Level 5 mit einer Remote-Control durch eine Art Lotsen, der mehrere Lkw in einem Rechenzentrum überwacht. Diese Lkw müssen nicht mehr einen Parkplatz an der Autobahn anfahren. Ermöglicht würde auf diese Weise zudem eine gewisse Entzerrung der Verkehrsströme, die nicht mehr an die Ruhezeiten gebunden wären, wodurch auch Off-PeakZeiten besser nutzbar wären. Ein solches Modell wäre selbstverständlich auch eine effektive Maßnahme zur Lösung des Fahrermangelproblems, das mit den heute diskutierten Ansätzen des Platoonings kaum wirksam adressiert werden kann.

\section{Fazit zum geplanten EU-Entsenderecht}

Die als Ergebnis des Trialogprozesses von EU-Kommission, EU-Parlament und Europäischem Rat auf EU-Ebene geplanten Regelungen des Entsenderechts und der Kabotage für den internationalen Straßengüterverkehr sind geeignet, Dienstleistungsfreiheit und Wettbewerb im Verkehr nachhaltig zu beschränken und protektionistischen Tendenzen in erheblichem Maße Vorschub zu leisten. Dies wird dem Publikum fälschlicherweise als Kampf gegen „Lohndumping“ und „unfaire“ Wettbewerbsbedingungen verkauft. Das Entsenderecht im Straßenverkehr sollte jedoch so ausgestaltet werden, dass es den freien Waren- und Dienstleistungsverkehr zwischen den Mitgliedstaaten und den freien Wettbewerb der Transportdienstleister nicht behindert und Diskriminierungstatbestände vermeidet bzw. minimiert. Die entsprechenden Vorschriften sind lediglich auf Kabotagefahrten anzuwenden, die keine (direkten) Auswirkungen auf den europäischen Güter- und Dienstleistungsverkehr haben. Als Referenzrahmen ist der nationale Mindestlohn, nicht aber ein nationaler oder sogar regionaler Branchentarif vorzusehen. Die Kabotageregelung sollte im Sinne des ursprünglichen Vorschlags der EU-Kommission vereinfacht werden (Fünf-Tages-Frist). Mittelfristig muss eine völlige Freigabe der Kabotage das Ziel sein.

Bei allen zu treffenden Regelungen ist auf eine Reduzierung der Bürokratiekosten zu achten, d.h., Nachweis- und Dokumentationspflichten sind auf ein Minimum zu begrenzen. Die EU-Vorschriften sollten so klar und deutlich formuliert werden, dass sie von nationalen Behörden nicht als Vorwand für Regelungen und Praktiken genutzt werden können, die Wettbewerber aus dem Ausland ohne sachlichen Grund diskriminieren und behindern. Es ist eine offene Frage, ob dies durch das rechtliche Vehikel der Richtlinie, deren Umsetzung weitgehend den Mitgliedstaaten überlassen bleibt, überhaupt erreichbar ist. Alle diese Forderungen erscheinen aber angesichts des Wechsels an der Kommissionspitze in weite Ferne gerückt. Die neue Kommissionspräsidentin hat bereits ihre sozialpolitische Agenda betont. Damit dürfte der Sicherstellung des Wettbewerbsprinzips im Güterkraftverkehr im EU-Binnenmarkt in Zukunft eine eher nachrangige Bedeutung zukommen.

Title: The Proposed EU Regulations for Posting Drivers in the Road Transport Sector - a Protectionist Affront?

Abstract: Road freight transport remains the principal mode of transport for the exchange of goods throughout the internal market, all political commitments in favour of a modal shift towards the railways notwithstanding. However, the road transport market appears to be increasingly subject to disruptions, particularly in view of the strong sectoral growth in recent years. The problem of an increasing shortage of drivers is being discussed in this context, as it is not only noticeable in Western Europe, but has begun to affect the Eastern European EU member states as well. Against this background, but more importantly, the question arises as to whether the principles of the internal market (especially the freedom to provide cross-border services) and the competitive situation in the national transport markets are still compatible. JEL Classification: F15, F23, F66, L92 